\title{
High-resolution ALMA Observations of HD 100546: Asymmetric Circumstellar Ring and Circumplanetary Disk Upper Limits
}

\author{
Jaime E. Pineda $^{1}$ (D), Judit Szulágyi ${ }^{2,3}$ (D) Sascha P. Quanz ${ }^{3,14}$ (D), Ewine F. van Dishoeck ${ }^{1,4}$ (iD), Antonio Garufi ${ }^{5,6}$ (D), \\ Farzana Meru $^{7}$ (D), Gijs D. Mulders ${ }^{8,13,15}$ (D), Leonardo Testi ${ }^{6,9}$ (D), Michael R. Meyer ${ }^{10,11}$ (D), and Maddalena Reggiani ${ }^{12}$ (iD \\ ${ }^{1}$ Max-Planck-Institut für extraterrestrische Physik, Giessenbachstrasse 1, D-85748 Garching, Germany; jpineda@mpe.mpg.de \\ ${ }^{2}$ Center for Theoretical Astrophysics and Cosmology, Institute for Computational Science, University of Zurich, Winterthurestrasse 190, CH-8057 Zurich, \\ Switzerland \\ ${ }^{3}$ Institute for Particle Physics and Astrophysics, ETH Zurich, Wolfgang Pauli Strasse 27, CH-8093 Zurich, Switzerland \\ ${ }^{4}$ Leiden Observatory, Leiden University, P.O. Box 9513, NL-2300 RA Leiden, The Netherlands \\ ${ }^{5}$ Universidad Autónonoma de Madrid, Dpto. Física Teórica, Módulo 15, Facultad de Ciencias, E-28049 Madrid, Spain \\ ${ }^{6}$ INAF/Osservatorio Astrofisico of Arcetri, Largo E. Fermi, 5, I-50125 Firenze, Italy \\ ${ }^{7}$ Institute of Astronomy, Madingley Road, Cambridge, CB3 OHA, UK \\ ${ }^{8}$ Lunar and Planetary Laboratory, The University of Arizona, 1629 E. University Boulevard., Tucson, AZ 85721, USA \\ ${ }^{9}$ European Southern Observatory, Karl-Schwarzschild-Str. 2, D-85748 Garching bei München, Germany \\ ${ }^{10}$ Institute for Astronomy, ETH Zurich, Wolfgang-Pauli-Strasse 27, 8093 Zurich, Switzerland \\ ${ }^{11}$ Department of Astronomy, University of Michigan, 500 Church Street, Ann Arbor, MI 48109, USA \\ ${ }^{12}$ Département d'Astrophysique, Géophysique et Océanographie, Université de Liège, 17 Allée du Six Août, B-4000, Liège, Belgium \\ ${ }^{13}$ Department of the Geophysical Sciences, The University of Chicago, Chicago, IL 60637, USA \\ Received 2018 July 6; revised 2018 November 16; accepted 2018 November 21; published 2019 January 21
}

\begin{abstract}
We present long-baseline Atacama Large Millimeter/submillimeter Array observations of the $870 \mu \mathrm{m}$ dust continuum emission and CO (3-2) from the protoplanetary disk around the Herbig Ae/Be star HD 100546, which is one of the few systems claimed to have two young embedded planets. These observations achieve a resolution of $4 \mathrm{au}$ (3.8 mas), an rms noise of $66 \mu \mathrm{Jy}_{\text {beam }^{-1}}$, and reveal an asymmetric ring between $\sim 20$ and 40 au with largely optically thin dust continuum emission. This ring is well fit by two concentric and overlapping Gaussian rings of different widths and a Vortex. In addition, an unresolved component is detected at a position consistent with the central star, which may trace the central inner disk ( $<2$ au in radius). We report a lack of compact continuum emission at the positions of both claimed protoplanets. We use this result to constrain the circumplanetary disk (CPD) mass and size of $1.44 M_{\oplus}$ and 0.44 au in the optically thin and thick regimes, respectively, for the case of the previously directly imaged protoplanet candidate at $\sim 55$ au (HD $100546 \mathrm{~b}$ ). We compare these empirical CPD constraints to previous numerical simulations. This suggests that HD $100546 \mathrm{~b}$ is inconsistent with several planet accretion models, while gas-starved models are also still compatible. We estimate the planetary mass as $1.65 M_{J}$ using the relation between planet, circumstellar, and circumplanetary masses derived from numerical simulations. Finally, the CO-integrated intensity map shows a possible spiral arm feature that could match the spiral features identified in near-infrared scattered light polarized emission, which suggests a real spiral feature in the disk surface that needs to be confirmed with further observations.
\end{abstract}

Key words: planet-disk interactions - protoplanetary disks - stars: formation - stars: individual (HD 100546) stars: pre-main sequence - techniques: interferometric

\section{Introduction}

Gas-rich and dust-rich disks around young stars are the birthplaces of new planetary systems. However, we still lack observational data that show under which physical and chemical conditions gas-giant planet formation takes place. Radial velocity (RV) exoplanet surveys have shown that $6 \%-7 \%$ of solar-type stars host gas-giant planets in the inner few au, and that the occurrence rate of these planets increases with stellar mass (Cumming et al. 2008; Johnson et al. 2010; Wittenmyer et al. 2016). Combining RV data with high-contrast imaging follow-up, Bryan et al. (2016) suggested that the total occurrence rate of companions with masses from 1 to $20 M_{\text {Jupiter }}$ and separations from 5 to 20 au could be as high as $\approx 50 \%$. In contrast, high-contrast direct imaging surveys reveal that beyond $50 \mathrm{au}$, massive giant planets are very rare (e.g., Lafrenière et al. 2007;

\footnotetext{
${ }^{14}$ National Center of Competence in Research "PlanetS" (http://nccrplanets.ch).

${ }^{15}$ Earths in Other Solar Systems Team, NASA Nexus for Exoplanet System Science.
}

Chauvin et al. 2010; Heinze et al. 2010; Biller et al. 2013; Nielsen et al. 2013; Rameau et al. 2013a; Wahhaj et al. 2013; Chauvin et al. 2015; Meshkat et al. 2015; Reggiani et al. 2016). However, planets of a few $M_{J}$ have been directly imaged around a few stars at orbital separations between 10 and 70 au (e.g., HR 8799, $\beta$ Pictoris, HD 95086, 51 Eri, HIP 65426; Marois et al. 2008; Lagrange et al. 2010; Rameau et al. 2013b; Macintosh et al. 2015; Chauvin et al. 2017).

On the theoretical side, there are two main theories for gasgiant planet formation: the core accretion (CA) paradigm (e.g., Pollack et al. 1996) and the gravitational instability (GI) theory (e.g., Boss 2001). The former, which is based on the initial growth of solids to eventually form the cores of gas-giant planets, has recently been modified to allow for a more rapid accretion of centimeter-sized and decimeter-sized particles (pebble accretion; Lambrechts \& Johansen 2012). It is unknown which of the mechanisms is responsible for the observed giant planet population or whether all of them contributed in different amounts (see Helled et al. 2014, for a recent review). 
To address these fundamental issues it is crucial to detect and study young giant planets in their formation phase, when they are still embedded in their natal environments. An elegant way to investigate the formation mechanism is to study the properties of the circumplanetary disk (CPD) that surrounds the young planet and transports material from the circumstellar disk (CSD) onto the forming object. CPD properties have been shown to be strongly dependent on the planet formation mechanism (Szulágyi et al. 2017). While analytic and numerical simulations generally agree that, irrespective of the formation mechanism, the CPD radius should be a fraction of the planet's Hill radius (Quillen \& Trilling 1998; Ayliffe \& Bate 2012; Shabram \& Boley 2013), their masses and temperatures are expected to be significantly different, with GI leading to more massive but colder CPDs compared to CA (Szulágyi et al. 2017). Hence, the direct detection of emission from CPDs, shedding light on their size and mass, would be a major step in understanding how gas-giant planets are formed.

Up to now, a few systems have shown direct evidence, based on high-contrast imaging observations, of candidate gas-giant planets that are still in their formation phase: HD 100546, which is the subject of this paper (see details on the system below), LkCa15 (Kraus \& Ireland 2012; Sallum et al. 2015), HD 169142 (Biller et al. 2014; Reggiani et al. 2014), MWC 758 (Reggiani et al. 2018), and PDS70 (Keppler et al. 2018). Isella et al. (2014) searched for CPD dust continuum emission in LkCa15 with the VLA, but did not succeed. For HD 100546 and HD 169142, the very red near-infrared (NIR) colors of the companion candidates are inconsistent with pure photospheric emission of young gas-giant planets, which led to the suggestion that the observed fluxes are a superposition of emission from a young planet and an additional CPD (Reggiani et al. 2014; Quanz et al. 2015). More recently, for HD 100546 $\mathrm{b}$, the emission from the CPD was predicted to be $800 \mu \mathrm{Jy}$ at $870 \mu \mathrm{m}$ (Zhu et al. 2016). Here, we present an analysis of new Atacama Large Millimeter/submillimeter Array Cycle 3 observations of the $870 \mu \mathrm{m}$ dust continuum emission of HD 100546 reaching an rms noise of $66 \mu \mathrm{Jy} \mathrm{beam}^{-1}$, and with high enough angular resolution to separate the CPD and CSD.

\section{The HD 100546 System}

HD 100546 is a Herbig Ae/Be star located at a distance of $110.02 \pm 0.62 \mathrm{pc}$ (Gaia Collaboration et al. 2018). The transition disk around this star has a cavity (in dust and molecular gas) between $~ 1-14$ au (e.g., Bouwman et al. 2003; Liu et al. 2003; Grady et al. 2005; Brittain et al. 2009; van der Plas et al. 2009; Benisty et al. 2010; Quanz et al. 2011; Liskowsky et al. 2012; Mulders et al. 2013; Panić et al. 2014; Sissa et al. 2018). The major axis is located at $145.14 \pm 0.04$ east of north (Pineda et al. 2014). The presence of a companion (HD 100546 c) inside this cavity was suggested by various studies based on both indirect and direct evidence (e.g., Bouwman et al. 2003; Acke \& van den Ancker 2006; Tatulli et al. 2011; Brittain et al. 2013; Mulders et al. 2013). However, Fedele et al. (2015) put forward the explanation that the spectroastrometric signature seen in the rovibrational $\mathrm{CO}$ emission lines (Brittain et al. 2013) does not require a planet, and Follette et al. (2017) claimed that the uncertain direct imaging detection from Currie et al. (2015) is caused by aggressive data processing. An additional protoplanet candidate (HD 100546 b) was identified further out in the outer disk ( 50-60 au separation from the central star) using high-contrast direct imaging observations (Quanz et al. 2013, 2015; Currie et al. 2014, 2017). However, this detection was called into question because no accretion features were detected (Rameau et al. 2017).

The current best dust continuum data published for HD 100546 are from ALMA C0 at $870 \mu \mathrm{m}$ (Pineda et al. 2014; Walsh et al. 2014) with 0". 6 resolution, and from the Australia Telescope Compact Array at $7 \mathrm{~mm}$, with an angular resolution of $0 . " 15$ (Wright et al. 2015). Both analyses of the ALMA C0 data (Pineda et al. 2014; Walsh et al. 2014) identified (in the uv-space) a ring-like structure of the dust emission that is more compact than the gas, while Walsh et al. (2014) also identified a second fainter ring farther out. However, the main discrepancy between these two works is the claim of an asymmetry in the dust continuum emission based on the residuals from the comparison of the best-fit model with the data by Pineda et al. (2014), while Walsh et al. (2014) claimed that their emission is symmetric based on the analysis of the interferometric visibilities. On the other hand, Wright et al. (2015) claimed an asymmetry at 7 and $3 \mathrm{~mm}$ in the images, but in the opposite direction reported by Pineda et al. (2014). The presence of asymmetries has been revealed in protostellar disks (e.g., van der Marel et al. 2013; Pérez et al. 2014; Casassus et al. 2015; Kraus et al. 2017), and has implications on the planet formation mechanism at play and their related timescales (e.g., Lyra \& Lin 2013; Mittal \& Chiang 2015). Therefore, and in order to search for direct evidence for CPDs, data with higher angular resolutions and image fidelity were needed to settle this issue.

\subsection{Updated Stellar Parameters}

The most up-to-date and accurate distance estimate (110.02 pc from GAIA DR2) to the star is larger than the previously derived estimate (97 pc from Hipparcos), which was used to estimate the stellar parameters, therefore we refine the stellar parameters based on $d=110.02 \mathrm{pc}$. We adopt a PHOENIX model of the stellar photosphere (Hauschildt et al. 1999) with $T_{\text {eff }}=9800 \mathrm{~K}$ (Fairlamb et al. 2015) and $\log (g)=$ -4.0 , then it is scaled to the GAIA DR2 distance and to the dereddened $\left(A_{\mathrm{V}}=0.1 \mathrm{mag}\right) V$-band photometry. The integrated luminosity $L_{*}$ is calculated from the model, which, combined with the aforementioned $T_{\text {eff }}$, are compared to the pre-main sequence stellar tracks by Siess et al. (2000). We employed the tracks with a depleted abundance of $Z$, because the source is depleted in refractory elements in its atmosphere (Folsom et al. 2012). This procedure yields a stellar mass and age of $M_{*}=$ $2.2 \pm 0.2 M_{\odot}$ and $t=4.8_{-1.1}^{+2.0} \mathrm{Myr}$, respectively. The reported uncertainties are obtained by propagating the uncertainties on the distance, $A_{\mathrm{V}}( \pm 0.1)$, and $T_{\text {eff }}$ (a conservative $\left.\pm 400 \mathrm{~K}\right)$.

\section{Data}

HD 100546 was observed on 2015 December 2 with ALMA using Band 7 receivers under project 2015.1.00806.S (PI: J.E. Pineda). The array configuration included 36 antennas with baselines between 17 and $10800 \mathrm{~m}$, but with insufficient short baselines $(<100 \mathrm{~m})$ to properly recover emission at scales larger than $\approx 1^{\prime \prime}$. The observations cycled through HD 100546 and quasar J1147-6753 with a cycle time of $\sim 1$ minute. The bright quasar J1427-4206 was used as a bandpass calibrator, while J1107-4449 was used to set the flux amplitude. The standard flagging and calibration were done using CASA 4.5.1 


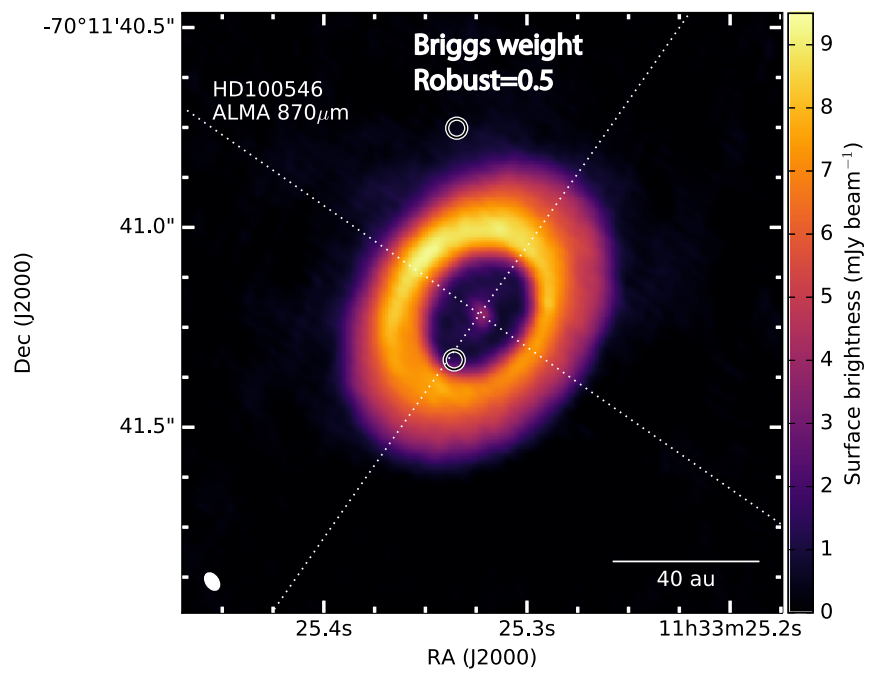

Figure 1. Synthesized image of the $870 \mu \mathrm{m}$ continuum emission from the HD 100546 disk using Briggs robust weight of 0.5 , with an $\mathrm{rms}$ of $66 \mu \mathrm{Jy}$ beam $^{-1}$ and a beam of $47 \times 31$ mas. Beam size and scale bar are shown in the bottom left and right corners, respectively. The markers show the positions of the claimed planets in the system. The dotted lines show the direction of the disk major and minor axes.

(McMullin et al. 2007), while imaging was done using CASA 4.7 and multiscale clean. Self-calibration was performed with the shortest phase and amplitude cycle of 10 and $60 \mathrm{~s}$, respectively. The $870 \mu \mathrm{m}$ continuum was obtained from linefree channels and imaged using natural weighting to achieve an angular resolution of $00^{\prime \prime} 056 \times 00^{\prime \prime} 041\left(\mathrm{PA}=26^{\circ} .9\right)$, with an rms noise of $86 \mu \mathrm{Jy}_{\text {beam }}{ }^{-1}$, as estimated from emission-free regions. Similarly, we imaged the continuum using a Briggs weight of 0.5 , which results in an angular resolution of $0{ }^{\prime \prime} 047 \times 0$ ". 031 $\left(\mathrm{PA}=33^{\circ} .9\right)$, and an rms noise of $66 \mu \mathrm{Jy}_{\text {beam }}{ }^{-1}$, as estimated from emission-free regions. Figure 1 shows the map using Briggs weight. A summary of the main observational parameters are listed in Table 1.

The total integrated flux of the image is 1.27 and $1.29 \mathrm{Jy}$ for the robust and naturally weighted images, respectively. This flux is consistent with the total flux measured in the ALMA C0 data.

We use the naturally weighted image when studying the CSD structure, while we use the image with robust weighting when investigating the existence of CPDs.

The CO (3-2) data cube is obtained from the continuumsubtracted visibilities resulting from using the task uvcontsub, after applying the self-calibration solutions obtained from the continuum. The imaging is done using a multiscale clean with natural weighting, which produced a beam size of 0 ."059 $\times 0 . \prime 044(\mathrm{PA}=18.22)$. Natural weighting is used, because it provides the highest sensitivity to spectral line observations. We estimate the rms in the spectral cube using the line-free channels as $5.8 \mathrm{mJy}^{-1}$ beam $^{-1}$ per channel, with a channel width of $0.209 \mathrm{~km} \mathrm{~s}^{-1}$ and a spectral resolution of two channels. In this case, the clean mask is defined for each channel around the bright emission; however, some imaging artifacts are still present due to the missing short spacings.

We use a Keplerian mask to calculate the moment maps, which is a similar to Friesen et al. (2017), Bergner et al. (2018), and Calcutt et al. (2018), where the region used in the calculation is limited to voxels (3D pixels) close to the emission. In order to create the mask, we calculate the predicted Keplerian velocity at
Table 1

Observational Parameters

\begin{tabular}{|c|c|c|}
\hline Parameter & Unit & Value \\
\hline \multicolumn{3}{|c|}{ Phase Center } \\
\hline R.A. & (hh:mm:ss.sss) & $11: 33: 25.318652$ \\
\hline Decl. & (dd:mm:ss.sss) & $-70: 11: 41.23173$ \\
\hline \multicolumn{3}{|c|}{ Continuum (Briggs weighting, Robust $=0.5$ ) } \\
\hline Wavelength & $(\mu \mathrm{m})$ & 870 \\
\hline Peak Flux & $\left(\mathrm{Jy} \mathrm{beam}^{-1}\right)$ & 9.27 \\
\hline Total Flux & (Jy) & 1.27 \\
\hline Beam Major axis & $(\operatorname{arcsec})$ & 0.047 \\
\hline Beam Minor axis & $(\operatorname{arcsec})$ & 0.031 \\
\hline Beam PA & $\left(^{\circ}\right)$ & 33.9 \\
\hline rms & $\left(\mu \mathrm{Jy} \mathrm{beam}^{-1}\right)$ & 66 \\
\hline \multicolumn{3}{|c|}{ CO (3-2) (natural weighting) } \\
\hline Beam Major axis & $(\operatorname{arcsec})$ & 0.059 \\
\hline Beam Minor axis & $(\operatorname{arcsec})$ & 0.044 \\
\hline Beam PA & $\left({ }^{\circ}\right)$ & 18.22 \\
\hline channel width & $\left(\mathrm{km} \mathrm{s}^{-1}\right)$ & 0.209 \\
\hline $\mathrm{rms}$ & $\left(\mathrm{mJy}\right.$ beam $^{-1}$ channel $^{-1}$ ) & 5.8 \\
\hline
\end{tabular}

each pixel, where we assume the stellar parameters derived in Section 2.1 and the disk parameter inclination and position angle derived from the continuum fit (see Table 2) and a disk radius of 352 au to match the extension of the $\mathrm{CO}$ emission as seen in the Cycle0 observations. The velocity field is then convolved with the same beam of the $\mathrm{CO}$ observations. Finally, only voxels that are within $2 \mathrm{~km} \mathrm{~s}^{-1}$ (similar to the line width in the inner part of the disk, $<150 \mathrm{au}$ ) from the predicted Keplerian velocity are kept in the final mask. The resulting integrated intensity map using the described mask is shown in Figure 2.

The total flux of the integrated intensity $\mathrm{CO}$ cube is $190 \mathrm{Jy} \mathrm{km} \mathrm{s}^{-1}$, which is in excellent agreement with the total integrated intensity $\mathrm{CO}$ reported by Panić \& Hogerheijde (2009) using APEX of $191 \mathrm{Jy} \mathrm{km} \mathrm{s}^{-1}$.

\section{Results \\ 4.1. Maps and Brightness Profile}

The maps shown in Figure 1 reveal a bright ring between 20 and 40 au with a significant flux asymmetry, and an additional inner disk coincident with the position of the star. The inner disk is unresolved ( $<2$ au in radius), with a peak flux of $2.60 \pm 0.85 \mathrm{mJy}$ beam $^{-1}$. Between the inner and outer disk there is a dark annulus with an average brightness of $\sim 1 \mathrm{mJy}$ beam ${ }^{-1}$, which is about $8 \times$ fainter than the (faintest section of the) central annulus of the ring emission.

In Figure 3 we compare the azimuthally averaged brightness temperature of the disk emission, for which we have calculated the deprojected radius using the position angle and disk inclination parameters obtained by Pineda et al. (2014). The same figure includes the parametric disk temperature profile from Panić et al. (2014) and the temperature profile of the millimeter-sized grains from the radiative transfer model from Pineda et al. (2014) (similar values of the midplane dust temperature at 50 au $(\approx 60 \mathrm{~K})$ were found by Bruderer et al. 2012). The figure shows that at every position in the disk the parametric disk temperature from Panić et al. (2014) is much higher than the observed values. However, the more detailed radiative transfer model reveals lower 
Table 2

Best-fit Parameters

\begin{tabular}{|c|c|c|c|}
\hline Parameter & Unit & Meaning & Value \\
\hline \multicolumn{4}{|c|}{ System Geometry } \\
\hline$\delta$ R.A. & $\left(10^{-3} \operatorname{arcsec}\right)$ & R.A. Offset from phase center & 20.6 \\
\hline$\delta$ Decl. & $\left(10^{-3} \operatorname{arcsec}\right)$ & Decl. Offset from phase center & 12.2 \\
\hline PA & (degrees) & Paralactic angle of the model ${ }^{\mathrm{a}}$ & 139.1 \\
\hline incl & (degrees) & Inclination of the model $^{\mathrm{b}}$ & 42.46 \\
\hline \multicolumn{4}{|c|}{ Ring \#1 } \\
\hline$F_{r}$ & $\left(\mathrm{Jy} \operatorname{arcsec}^{-2}\right)$ & Ring peak surface brightness & 1.50 \\
\hline \multirow[t]{2}{*}{$r_{v}$} & $(\operatorname{arcsec})$ & Ring radius & 0.186 \\
\hline & (au) & Ring radius & 20.5 \\
\hline \multirow[t]{2}{*}{$\sigma_{v}$} & $(\operatorname{arcsec})$ & Ring width & 0.0303 \\
\hline & (au) & Ring width & 3.33 \\
\hline \multicolumn{4}{|c|}{ Ring \#2 } \\
\hline$F_{r 2}$ & $\left(\mathrm{Jy} \operatorname{arcsec}^{-2}\right)$ & Ring peak surface brightness & 4.38 \\
\hline \multirow[t]{2}{*}{$r_{v 2}$} & $(\operatorname{arcsec})$ & Ring radius & 0.270 \\
\hline & (au) & Ring radius & 29.7 \\
\hline \multirow[t]{2}{*}{$\sigma_{v 2}$} & $(\operatorname{arcsec})$ & Ring width & 0.0919 \\
\hline & (au) & Ring width & 10.1 \\
\hline \multicolumn{4}{|c|}{ Vortex } \\
\hline$F_{v}$ & $\left(\mathrm{Jy}_{\operatorname{arcsec}}{ }^{-2}\right)$ & Vortex peak surface brightness & 1.31 \\
\hline \multirow[t]{2}{*}{$r_{v}$} & $(\operatorname{arcsec})$ & Vortex radius & 0.198 \\
\hline & (au) & Vortex radius & 21.8 \\
\hline \multirow[t]{2}{*}{$\sigma_{v}$} & $(\operatorname{arcsec})$ & Vortex width & 0.0804 \\
\hline & (au) & Vortex width & 8.85 \\
\hline$\theta_{v}$ & (degree) & Vortex position angle ${ }^{c}$ & -88.6 \\
\hline$\sigma_{v, \theta}$ & (degree) & Vortex angular width & 44.6 \\
\hline \multicolumn{4}{|c|}{ Central Inner Disk } \\
\hline$F_{g}$ & (mJy) & Gaussian flux & 8.50 \\
\hline$\sigma_{g}$ & $\left(10^{-3} \operatorname{arcsec}\right)$ & Gaussian width & 5.59 \\
\hline$\Delta x_{g}$ & $\left(10^{-3} \operatorname{arcsec}\right)$ & Offset along deprojected $x$-axis & -23.1 \\
\hline$\Delta y_{g}$ & $\left(10^{-3} \operatorname{arcsec}\right)$ & Offset along deprojected $y$-axis & -7.19 \\
\hline
\end{tabular}

Notes.

${ }^{\mathrm{a}}$ Measured due east from north.

b A value of $0^{\circ}$ is face on, and $90^{\circ}$ is edge-on.

${ }^{c}$ Measured due east from the system position angle.

temperatures for the millimeter-sized particles, with an average temperature of $T_{d \text {,mean }}=53 \mathrm{~K}$ between 20 and $50 \mathrm{au}$. We use this average disk dust temperature, $T_{d \text {,mean }}$, as a best estimate of the disk emission in the following sections. Therefore, the disk dust continuum emission is optically thin at the positions of the young planet candidates, while the central part of the ring might be optically thick.

\subsection{Parametric Model}

We model the emission with a simple parametric model that includes two Gaussian rings, a central compact source, and a vortex (to account for flux asymmetries), as follows:

$$
\begin{aligned}
F\left(r, r_{1}, \theta\right)= & F_{r 1} e^{-\left(r-r_{r 1}\right)^{2} / 2 \sigma_{r 1}^{2}} \\
& +F_{r 2} e^{-\left(r-r_{r 2}\right)^{2} / 2 \sigma_{r 2}^{2}} \\
& +F_{g} e^{-r_{p}^{2} / 2 \sigma_{g}^{2} /\left(2 \pi \sigma_{g}^{2}\right)} \\
& +F_{V} e^{-\left(r-r_{v}\right)^{2} /\left(2 \sigma_{v}^{2}\right)} e^{-\left(\theta-\theta_{v}\right)^{2} /\left(2 * \sigma_{\theta}^{2}\right)}
\end{aligned}
$$

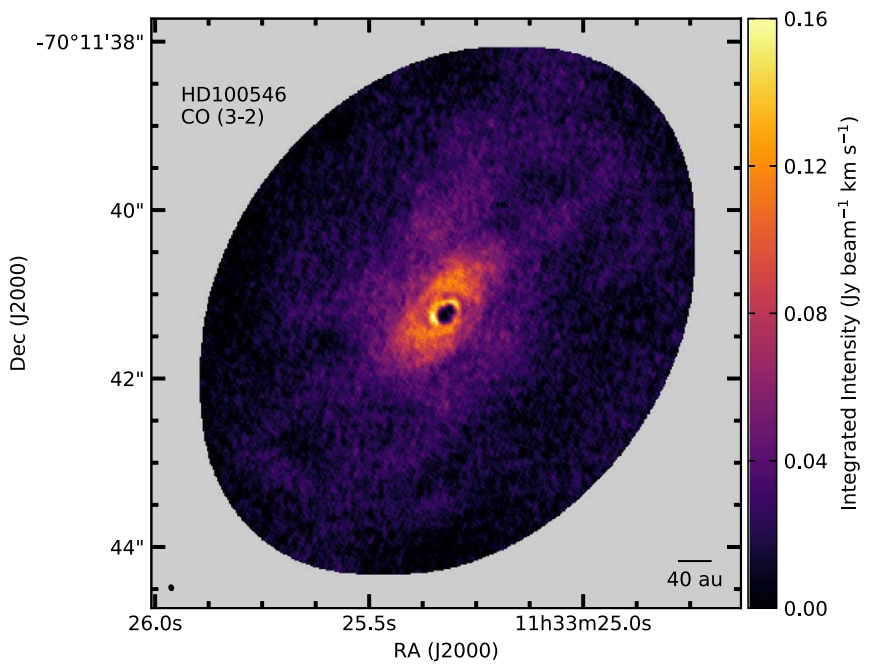

Figure 2. Integrated intensity map of high-resolution $\mathrm{CO}(3-2)$ emission for the HD 100546 disk calculated using the Keplerian velocity mask. The field of view shown is larger than the continuum image shown in Figure 1. The beam size and scale bar are shown in the bottom left and right corners, respectively.

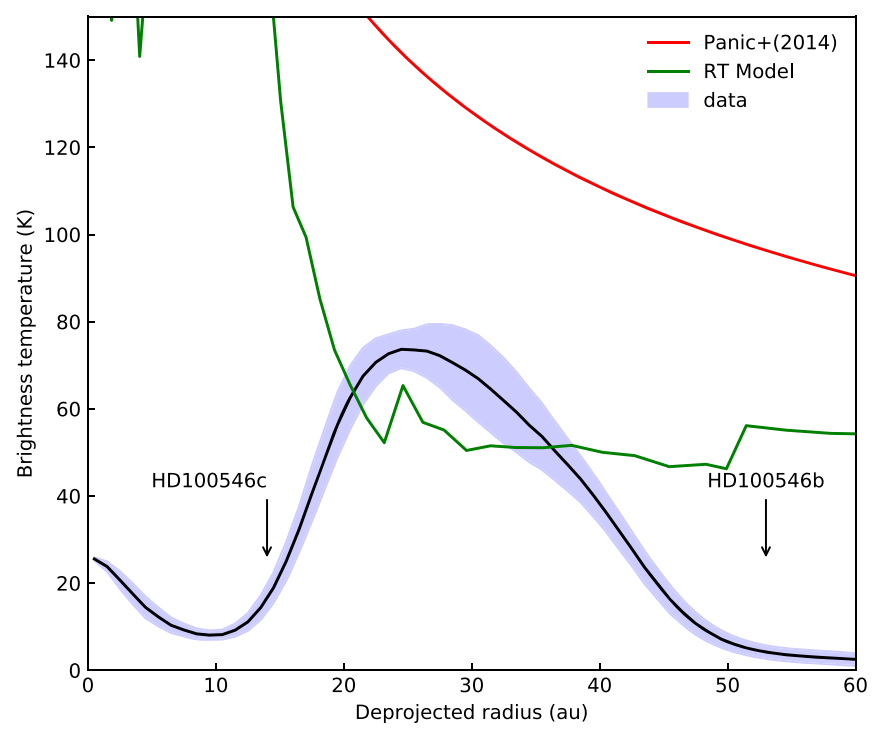

Figure 3. Azimuthally averaged brightness temperature profile (black line). The shaded area shows the local standard deviation of the measurements. The average is done on the deprojected disk geometry. The red curve is the temperature profile used by Panić et al. (2014), and the green curve is the temperature profile of the best radiative transfer model from Pineda et al. (2014). The vertical arrows mark the expected position of the two planet candidates at 14 and 53 au.

where $r$ and $r_{r}$ are the radii calculated at the center of the ring and point source, respectively, and taking into account the inclination angle with respect to the sky (assumed to be the same for both coordinate systems). The first two elements in the model attempt to reproduce the main disk ring-like emission (which is not well reproduced by a single Gaussian profile) and are concentric, the third one describes the central unresolved source, and the fourth element describes a possible vortex.

We use GALARIO (Tazzari et al. 2018) to sample the model image on the same visibilities as the observations. The $\chi^{2}$ is then calculated using the sampled visibilities, and then 

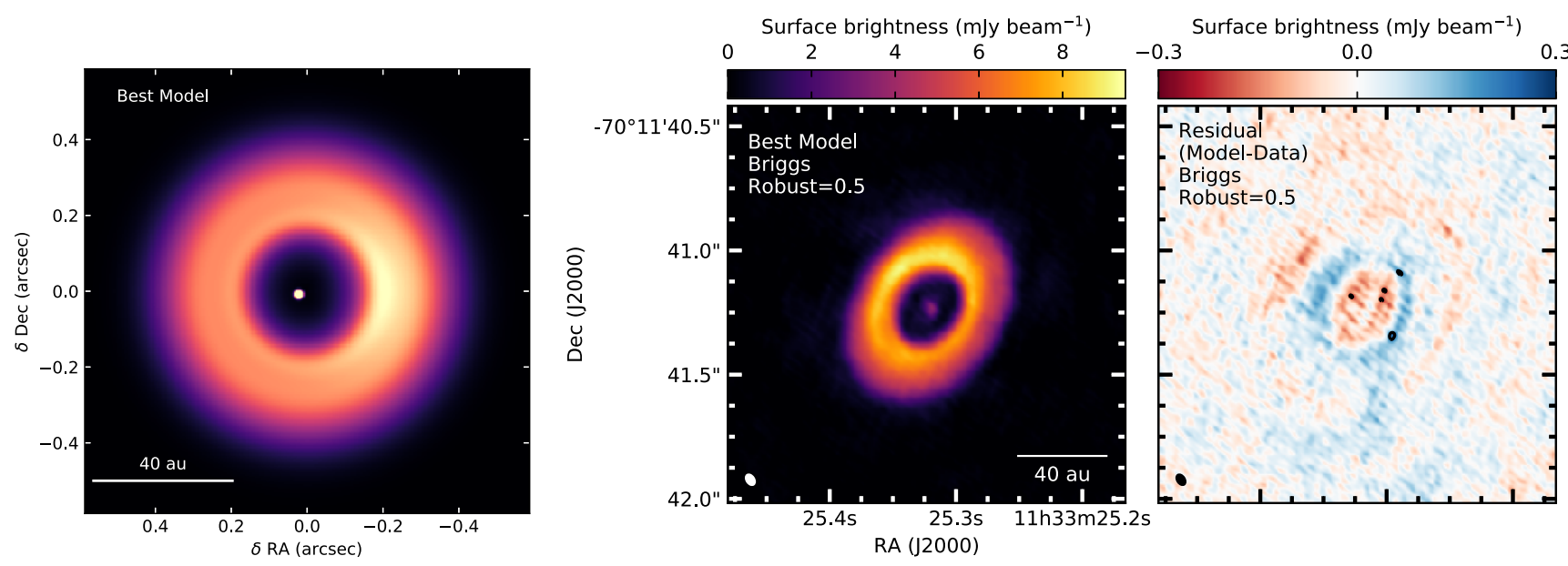

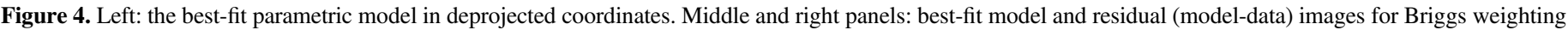

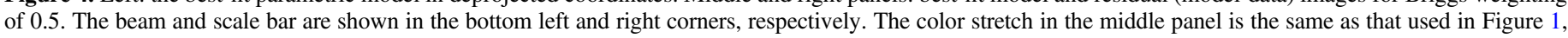

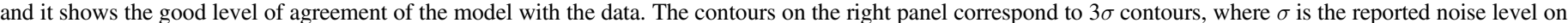
the observed map.

minimized in Python to find the optimal model. Also, we use the built-in options in GALARIO to perform 2D translations in the plane of the sky ( $\delta$ R.A., $\delta$ decl.) and rotation ( $\delta$ PA) of the parametric model. The best-fit model in deprojected coordinates, the observed model, and the residuals are shown in Figure 4, while the best parameter values are listed in Table 2. The observed model and residuals are produced by sampling the same visibilities as the data, and then performing the imaging in CASA.

The combined vortex and double-ring model allows for a good fit of the image, although the residuals still show some structure, in particular close to the ring inner edge. However, none of these two rings or vortex correspond to the outer ring found by Walsh et al. (2014). We also note that the best fit confirms what is seen by eye: a significant offset between the central Gaussian source and the central position of the ring.

\subsection{Radial Cuts}

We generate two cuts, one through the disk major axis and one through the vortex maximum emission to investigate in more detail the ring morphology. Figure 5 shows the average flux along beam-wide strips along both directions. The profiles can clearly not be fitted with a single Gaussian flux distribution and they show significant asymmetries in the peak flux on both sides $(\approx 15 \%-25 \%)$. Fitting the profiles with a superposition of five Gaussians provides a good fit, however. The best-fit parameters are summarized in Table 3.

We also attempted to fit the profiles with asymmetric Gaussians (see, e.g., Pinilla et al. 2017), but the results are rather poor and therefore not reported here.

Moreover, we decompose the deprojected map into its polar coordinates (radius and angle) to better understand the radial structure of the emission (see Figure 6). The plot confirms the radial asymmetry in the main ring, with a "slow" flux drop as the radius increases beyond the $\approx 30$ au radius. On the other hand, the ring emission has a steeper inner edge and clear azimuthal asymmetry. However, all these structures are unrelated to a previous outer ring claimed by Walsh et al. (2014) at $\approx 190 \mathrm{au}$.

\subsection{CPD Emission}

Numerical simulations predict the presence of CPDs around young forming gas-giant planets (e.g., Szulágyi et al. 2014, 2017; Zhu et al. 2016). The expected disk sizes are supposed to be much smaller than the beam size of the observations presented here $(\sim 3 \mathrm{au})$. Therefore, we expect any CPD emission to appear point-like in our data. However, we do not find any evidence for point-like emission close to or around the claimed protoplanet positions and place a strong $3 \sigma$ detection limit of $198 \mu \mathrm{Jy}$ for an unresolved source.

\subsection{CO Emission}

The CO (3-2) emission (Figure 7 ) extends out to $\approx 2 ! .7$ (300 au), which is less extended than the emission detected with the ALMA Cycle0 data (Pineda et al. 2014; Walsh et al. 2014) because of the missing short baselines in our observations. Clearly the $\mathrm{CO}$ emission is much more extended than the continuum emission, which was already identified in the $\mathrm{Cycle} 0$ analysis. The first-moment (intensity weighted velocity) map is presented in Figure 7, overlaid with the continuum emission. The position-velocity (PV) diagram along the disk's major axis is presented in Figure 8 . The Keplerian velocity profile for the HD 100546 system, with $M_{*}=2.2 M_{\odot}$ and $42^{\circ}$ inclination angle, reproduces the velocities at a distance $>2^{\prime \prime}$ from the star (red curve in Figure 8). For separations $<2^{\prime \prime}$, the velocities are better reproduced with an inclination angle of $32^{\circ}$ (orange curve in Figure 8).

\section{Discussion}

\subsection{CPD Upper Limits}

Given the non-detection of CPD emission toward HD $100546 \mathrm{~b}$, we place upper limits on the mass or size of the CPD, depending on the assumption of optically thin or thick emission.

In the case of the CPD emission being optically thin, we estimate the total CPD mass via

$$
M_{\mathrm{total}}=\frac{d^{2} F_{\nu}}{B_{\nu}\left(T_{d}\right) \kappa_{\nu} f_{d}},
$$




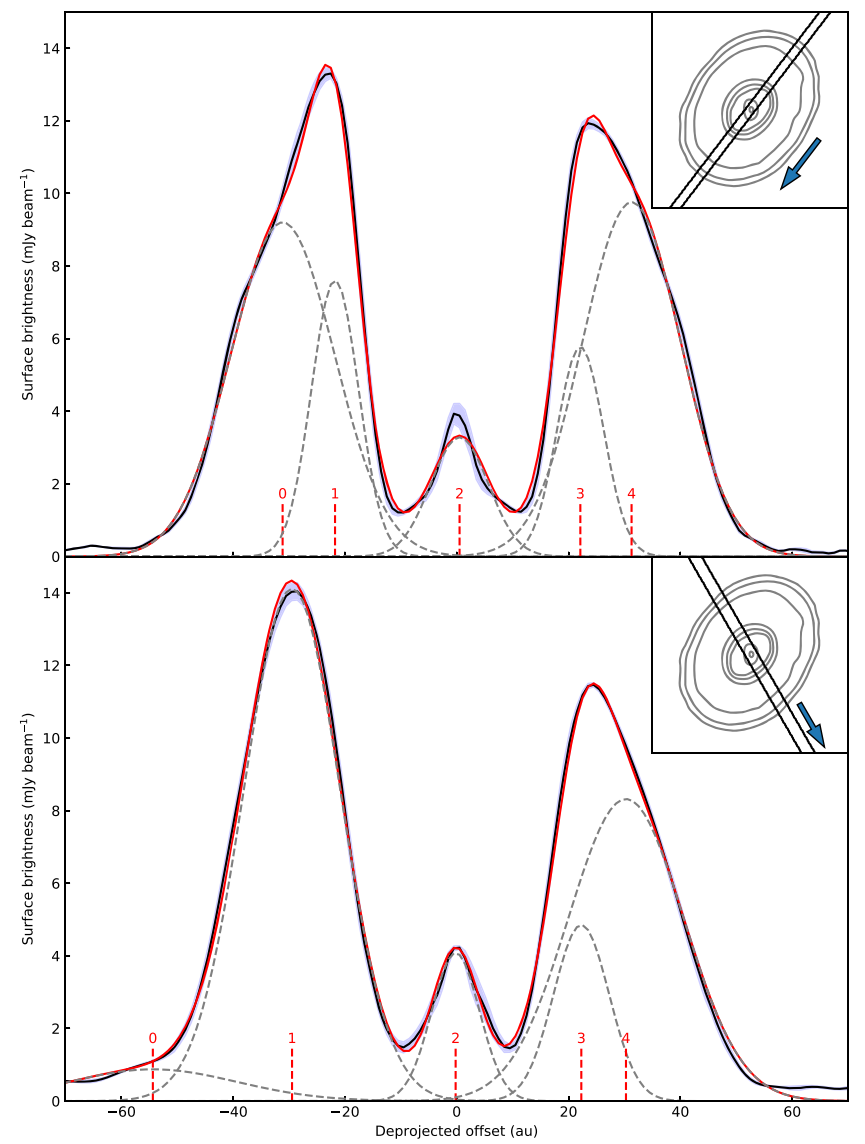

Figure 5. The top and bottom panels show radial cuts along the major axis and the vortex, respectively. The black lines are the average of the beam-wide strips. The profiles are clearly asymmetric and well reproduced by a superposition of five Gaussians. The dashed lines show the individual Gaussians (see, Table 3) and the red lines show their sum. An inset showing the orientation of the cuts is presented in the top right corners of each panel.

where $\kappa_{\nu}$ is the dust opacity per dust mass, $d$ is the distance to the source, $F_{\nu}$ is the observed flux, $B_{\nu}\left(T_{d}\right)$ is the blackbody function, and $f_{d}$ is the dust-to-gas ratio. For the opacity we assume $\kappa_{\nu}=0.2\left(7 \mathrm{~mm} \lambda^{-1}\right) \mathrm{cm}^{2} \mathrm{~g}^{-1}$, which is consistent with the value used by Isella et al. (2014). This opacity assumes a dust composition and grain size distribution as in Isella et al. (2012). We note that this $\kappa_{\nu}$ is a factor $\approx 2 \times$ lower than that used by Beckwith et al. (1990) and Andrews et al. (2011), and therefore our mass upper limits are conservative. Finally, $f_{d}$ is assumed to be 0.01. Given the emission upper limit determined in Section 4.4, we determine the CPD (dust and gas) mass upper limit in the optically thin case to be $1.44 M_{\oplus}$.

In the case of the CPD emission being optically thick, the disk radius is calculated from $F_{\nu}=B_{\nu}\left(T_{d}\right) \Omega$, where $T_{d}$ is the dust temperature of the CPD and $\Omega$ is the area subtended on the sky $\left(\Omega=\pi R_{\mathrm{CPD}}^{2} / d^{2}\right)$. Therefore, the radius is derived as

$$
R_{\mathrm{CPD}}=\sqrt{\frac{F_{\nu}}{\pi B_{\nu}\left(T_{d}\right)}} d .
$$

An upper limit for the CPD radius of 0.44 au is obtained using a CPD temperature equal to the mean dust temperature for the millimeter-sized particles in the radiative transfer model at that radius $\left(T_{d \text {,mean }}=53 \mathrm{~K}\right.$, see Section 4.1$)$, while the radius is

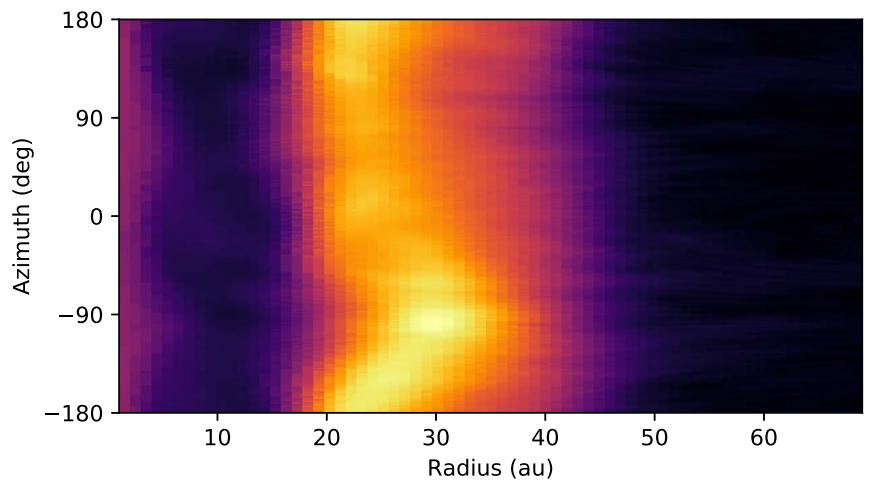

Figure 6. Deprojected continuum image in polar coordinates. The ring emission is non-Gaussian, as exemplified in Figure 5. The azimuthal angle is measured from north due east from the the disk semimajor axis, with $0^{\circ}$ in the southeast direction.

Table 3

Multiple-Gaussian Fit ${ }^{\mathrm{a}}$

\begin{tabular}{lcccc}
\hline \hline Component & $\begin{array}{c}\text { Center } \\
(\mathrm{au})\end{array}$ & $\begin{array}{c}\text { Peak Flux } \\
\left(\mathrm{mJy} \mathrm{beam}^{-1}\right)\end{array}$ & $\begin{array}{c}\sigma \\
(\mathrm{au})\end{array}$ \\
\hline \multicolumn{5}{c}{ NW-SE } \\
\hline$\# 0$ & $-31.1 \pm 0.7$ & $9.2 \pm 0.4$ & $8.9 \pm 0.3$ \\
$\# 1$ & $-21.7 \pm 0.2$ & $7.6 \pm 0.8$ & $3.3 \pm 0.4$ \\
$\# 2$ & $0.5 \pm 0.3$ & $3.3 \pm 0.2$ & $4.5 \pm 0.4$ \\
$\# 3$ & $22.1 \pm 0.2$ & $5.8 \pm 0.7$ & $3.2 \pm 0.5$ \\
$\# 4$ & $31.3 \pm 0.6$ & $9.8 \pm 0.3$ & $8.4 \pm 0.2$ \\
\hline \multicolumn{7}{c}{ NE-SW } \\
$\# 0$ & $-54.3 \pm 8.7$ & $0.88 \pm 0.09$ & $14.7 \pm 18.5$ \\
$\# 1$ & $-29.4 \pm 0.3$ & $14.11 \pm 0.96$ & $8.2 \pm 0.3$ \\
$\# 2$ & $-0.2 \pm 0.3$ & $4.08 \pm 0.08$ & $3.4 \pm 0.3$ \\
$\# 3$ & $22.3 \pm 0.2$ & $4.85 \pm 0.67$ & $4.2 \pm 0.5$ \\
$\# 4$ & $30.3 \pm 0.7$ & $8.32 \pm 0.39$ & $10.0 \pm 0.2$ \\
\hline
\end{tabular}

Note.

${ }^{\text {a }}$ Each Gaussian is described as:

$$
f(x)=F_{\text {peak }} e^{-\left(x-x_{\text {center }}\right)^{2} / 2\left(\sigma^{2}+\sigma_{\text {beam }}^{2}\right)}
$$

only $0.09 \mathrm{au}$ for a temperature of $932 \mathrm{~K}$, which is the estimated temperature from high-contrast imaging at the $L$ - and $M$-bands (Quanz et al. 2015). Both numbers are much smaller than the 2.8 au radius of the Hill sphere expected for a $1 M_{J}$ planet at $53 \mathrm{au}$ (HD 100546 b). Several studies have determined the CPD radius to be between 0.3 and 0.5 of the Hill radius (Quillen \& Trilling 1998; Ayliffe \& Bate 2012; Shabram \& Boley 2013) A conservative CPD radius' upper limit of $0.44 \mathrm{au}$ yields an upper limit for the planet mass of $47 M_{\oplus}\left(0.15 M_{J}\right)$.

Both cases, optically thin and thick limits, provide important constraints for gas-giant planet formation processes by constraining fundamental properties of CPDs. In addition, Zhu et al. (2016) provided predictions for the SEDs of CPDs, including fluxes up to the submillimeter regime based on the predicted flux at $870 \mu \mathrm{m}$, $\sim 800 \mu \mathrm{Jy}$, which is almost a factor of $10 \times$ the noise level in the image. On the other hand, detailed numerical simulations and synthetic observations of CPDs carried out by Szulágyi et al. (2018) showed that at large separations from the central star, a small fraction of the CPDs $\left(<R_{\mathrm{Hill}} / 3\right)$ are warmer than the CSD. 


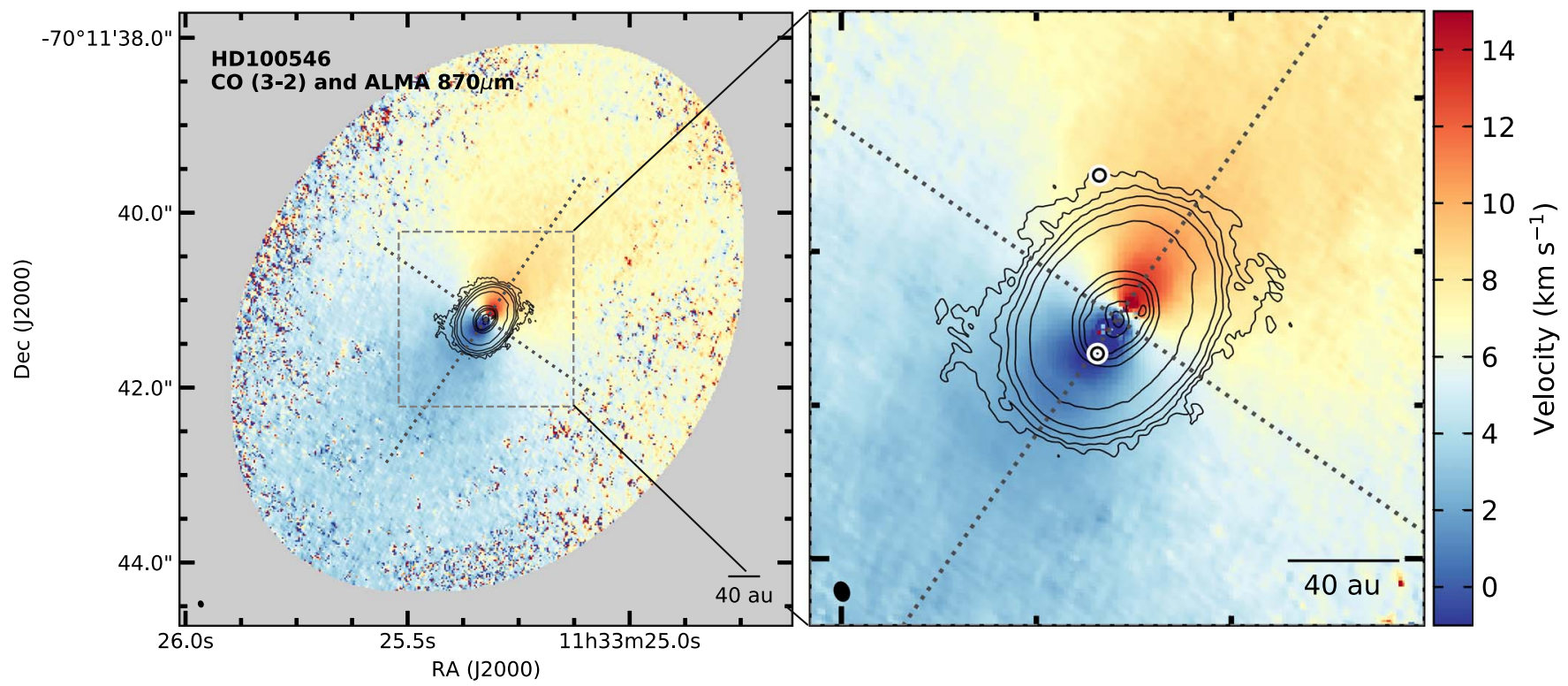

Figure 7. CO (3-2) first-moment map (centroid velocity) for the HD 100546 disk using the Keplerian velocity mask, shown in color. The $870 \mu \mathrm{m}$ continuum emission map, using robust Briggs weighting, is overlaid in contours shown at $[5,10,20, \ldots, 320] \times \mathrm{rms}$, where rms is $66 \mu \mathrm{Jy} \mathrm{beam}^{-1}$. The left panel shows the full disk emission, while the right panel shows a zoomed-in view of the region of the continuum emission. The dotted lines show the major and minor axes obtained from fitting the dust continuum visibilities. The circles show the positions of the two planet candidates for HD 100546. The synthetized beam is shown in the bottom left corner.

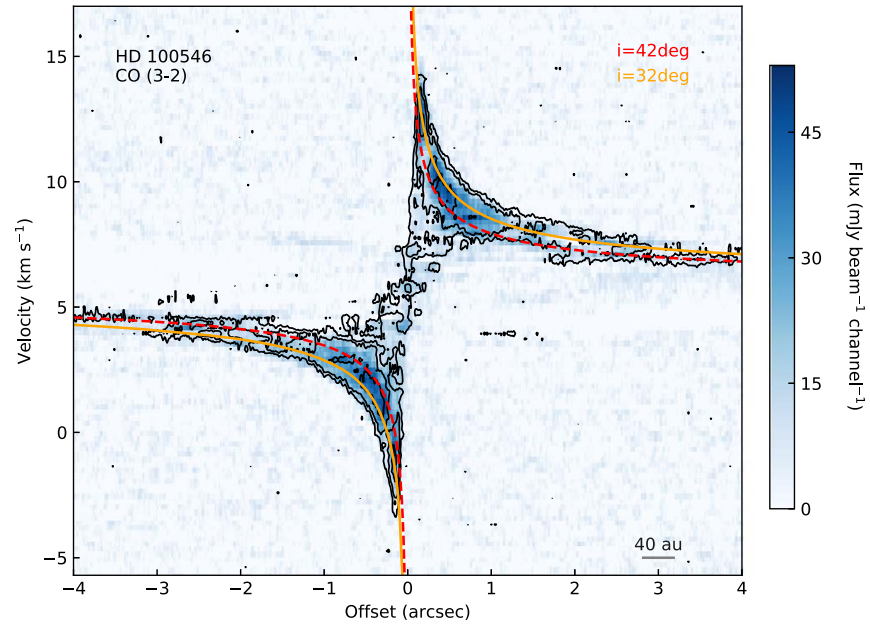

Figure 8. PV diagram of $\mathrm{CO}(3-2)$ along the major axis shown in Figure 7. Contours are shown at $[3,6,12] \times \mathrm{rms}$, where rms is $3.3 \mathrm{mJy}_{\text {beam }}{ }^{-1}$ per channel. The orange and red curves show the expected Keplerian velocity for a central star of $2.2 M_{\odot}$ and inclination angle of $32^{\circ}$ and $42^{\circ}$, respectively.

Furthermore, based on the nominal CSD setup used by Szulágyi et al. (2018) the expected flux for a CPD around a $1 M_{J}$ planet at $52 \mathrm{au}$ is $\approx 250 \mu \mathrm{Jy}$, which is comparable to the upper limits reported here, and therefore is still consistent with the ALMA observations.

According to de Val-Borro et al. (2007), even a Neptunemass planet can generate a vortex of Rossby-Wave Instability, so this is consistent with our planetary mass limit. How strong this vortex is depends on many factors apart from the planetary mass: dust-to-gas ratio, viscosity, magnetic field of the disk, etc. A detailed parameter study of various numerical simulations is needed for this system in order to constrain the planetary mass based on the vortex we observe, as has been done for IRS48 (Huang et al. 2018), which is beyond the scope of this work.

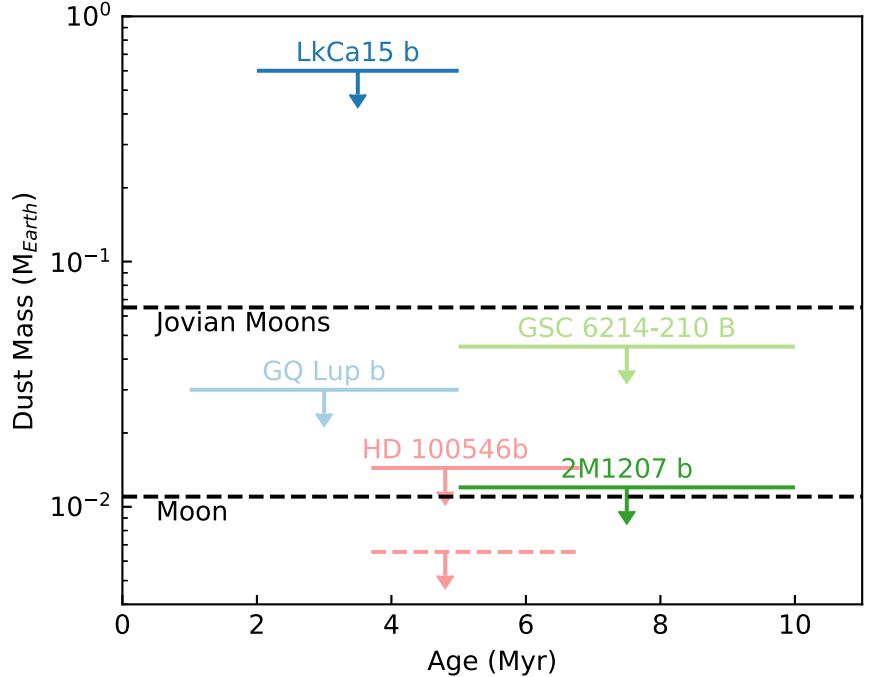

Figure 9. Adapted from Ricci et al. (2017). CPD mass upper limits are shown as a function of the central object's estimated age. For HD $100546 \mathrm{~b}$ we show two estimates: (1) the solid red bar shows the value reported in Section 4.4, and (2) the CPD mass when using the same dust opacity as for the other CPD estimates shown. We also show the mass contained in the Jovian moons and the Earth moon for comparison (dashed lines).

\subsection{CPD Masses and Ages}

Figure 9 compares the results of a few studies that have provided upper limits for CPD masses (see also, Ricci et al. 2017). The CPD mass upper limit obtained for HD $100546 \mathrm{~b}$ in Section 5.1 is comparable to that reported by Ricci et al. (2017); however, our assumed dust opacity is smaller, therefore we re-scale the CPD mass estimate to the one used by Ricci et al. (2017) and plotted it using the dashed line in Figure 9. This sample includes systems covering a wide range of stellar (host) mass and environments. However, it consistently shows that the CPDs, in case they do exist, carry only a small amount of mass. This is at odds with several models that generate 
substantial CPDs to feed protoplanets (Gressel et al. 2013; Shabram \& Boley 2013; Stamatellos \& Herczeg 2015; Zhu et al. 2016). On the other hand, the current CPD mass limits are consistent with the "gas-starved" disk scenario proposed by Canup \& Ward (2002, 2006), as well as the numerical simulations by Szulágyi (2017) that show a correlation between CPD mass and CSD mass.

We calculate an upper limit for the potential planet's mass using Equation (7) from Szulágyi (2017),

$$
M_{\mathrm{CPD}} \times 10^{4}=3.17 M_{\mathrm{CSD}} M_{p}-4.33 M_{\mathrm{CSD}},
$$

which relates the CPD, planetary $\left(M_{p}\right)$, and CSD mass (all in units of $M_{J}$ ). This assumes that the planet is still accreting from the surrounding CSD, which is supported by the previous detection of $L^{\prime}$ - and $M$-band thermal emission. Assuming the optically thin CPD estimate case from above, we place a planetary mass upper limit of $1.65 M_{J}$, using our CPD mass upper limit of $1.44 M_{\oplus}\left(0.0045 M_{J}\right)$ and the CSD mass of $50 M_{J}$ (Pineda et al. 2014). This upper limit on the planetary mass estimate is clearly less stringent than the one derived using the optically thick approximation in Section 5.1; however, the upper limit calculated using the relation between CPD and CSD does have a less strong assumption and might be more realistic than the one reported in Section 5.1.

\subsection{Central or Inner Disk}

The central emission is compact and represents the innermost circumstellar material. We fitted a single Gaussian over a 80 mas region with a total flux of $13.6 \pm 1.0 \mathrm{mJy}$, a deconvolved FWHM of the major and minor axis of $80 \pm 8$ mas and $56 \pm 6$ mas, respectively, and with a position angle of $177^{\circ} \pm 14^{\circ}$.

We use Equation (2), the same dust properties used in Section 5.1, and a disk temperature of $300 \mathrm{~K}$, to derive an inner disk mass of $15 M_{\oplus}$. The stellar accretion rate of the central star is estimated to be $\dot{M}_{*}=10^{-7.04_{-0.15}^{+0.13}} M_{\odot} \mathrm{yr}^{-1}$ (Fairlamb et al. 2015). Thus, the central disk depletion lifetime $\left(M_{\text {disk }} / \dot{M}\right)$ is only $500 \mathrm{yr}$. Therefore, the disk must be replenished with material from the outer ring/disk (e.g., Pinilla et al. 2016).

\subsection{Comparison with SPHERE Scattered Light Data}

Garufi et al. (2016) presented an unsharp masked version of the HD 100546 disk based on SPHERE/ZIMPOL polarimetric differential imaging data. This image shows the disk inner rim, a spiral to the NE, and an arm-like structure to the north. In Figure 10 we show the SPHERE $Q_{\phi}$ image with our ALMA continuum map overlaid in contours, while Figure 11 similarly compares it to the CO-integrated intensity. The SPHERE data are aligned to match the star position with the center of the compact dust continuum emission.

This comparison confirms that the disk inner rim is well-traced by the SPHERE observations and by the ALMA observations (continuum and $\mathrm{CO}$ ). The NE-spiral feature observed in the SPHERE data coincides with the central region of the ring in the continuum emission, which indicates that the spiral-like feature in scattered light does not have a counterpart in the midplane. However, this feature location and the general orientation are coincident with a spiral-like enhancement seen in the COintegrated intensity. This coincidence might suggest that the

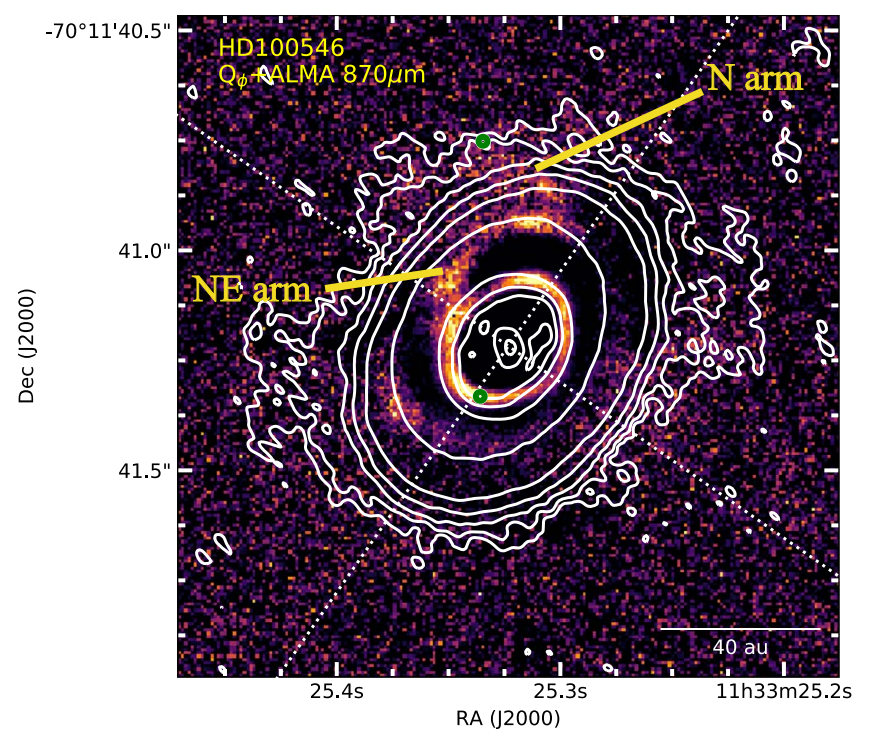

Figure 10. Unsharp masked version of a SPHERE/ZIMPOL $Q_{\phi}$ image overlaid with our ALMA continuum data (white contours). Marked are the spiral features identified from the SPHERE data. The NE-arm feature matches the central location of the ring-like continuum emission. The $\mathrm{N}$-arm feature is located close to the low-level brightness emission close to HD $100546 \mathrm{~b}$. The green markers show the position of the claimed planets in the system.

spiral-like feature is real and present in the disk surface. This is consistent with the fact that small dust grains and gas are well coupled in those disk regions.

\subsection{Disk Kinematics}

Based upon the low angular resolution CO (3-2) ALMA Cycle0 observations, a warp disk was claimed by Pineda et al. (2014) by comparing the PV diagram along the major axis. Since the stellar mass is well constrained, by overplotting the expected Keplerian curves it was clear that a single-disk inclination could not reproduce the observations (see Loomis et al. 2017, for a similar claim of a warp in AA Tau). Further analysis of the same data, but using a more complex modeling tool, also suggested the presence of a change in the disk inclination (Walsh et al. 2017). Our results also show a similar pattern in the centroid velocity map (Figure 7), where the inner disk region is slightly twisted.

The possible disk warp has been suggested before to explain different observations (Quillen 2006; Panić et al. 2010). Figure 8 shows the PV diagram along the disk major axis, which shows the same behavior seen from the previous low angular resolution, with the kinematics of the outer section of the disk $\left(>2^{\prime \prime}\right)$ being better described by an inclination angle of $\approx 42^{\circ}$, while the inner section of the disk $(<0$."5) is better described by an inclination angle closer to $\approx 32^{\circ}$. This means that the whole disk is not well described by a single inclination angle.

Also, it has been proposed that departures from the Keplerian velocity field in the disk kinematics could provide an independent way to identify the presence of a CPD in HD 100546 (Perez et al. 2015). Unfortunately, the image fidelity and sensitivity of the $\mathrm{CO}(3-2)$ data presented here do not allow us to identify such a feature. 


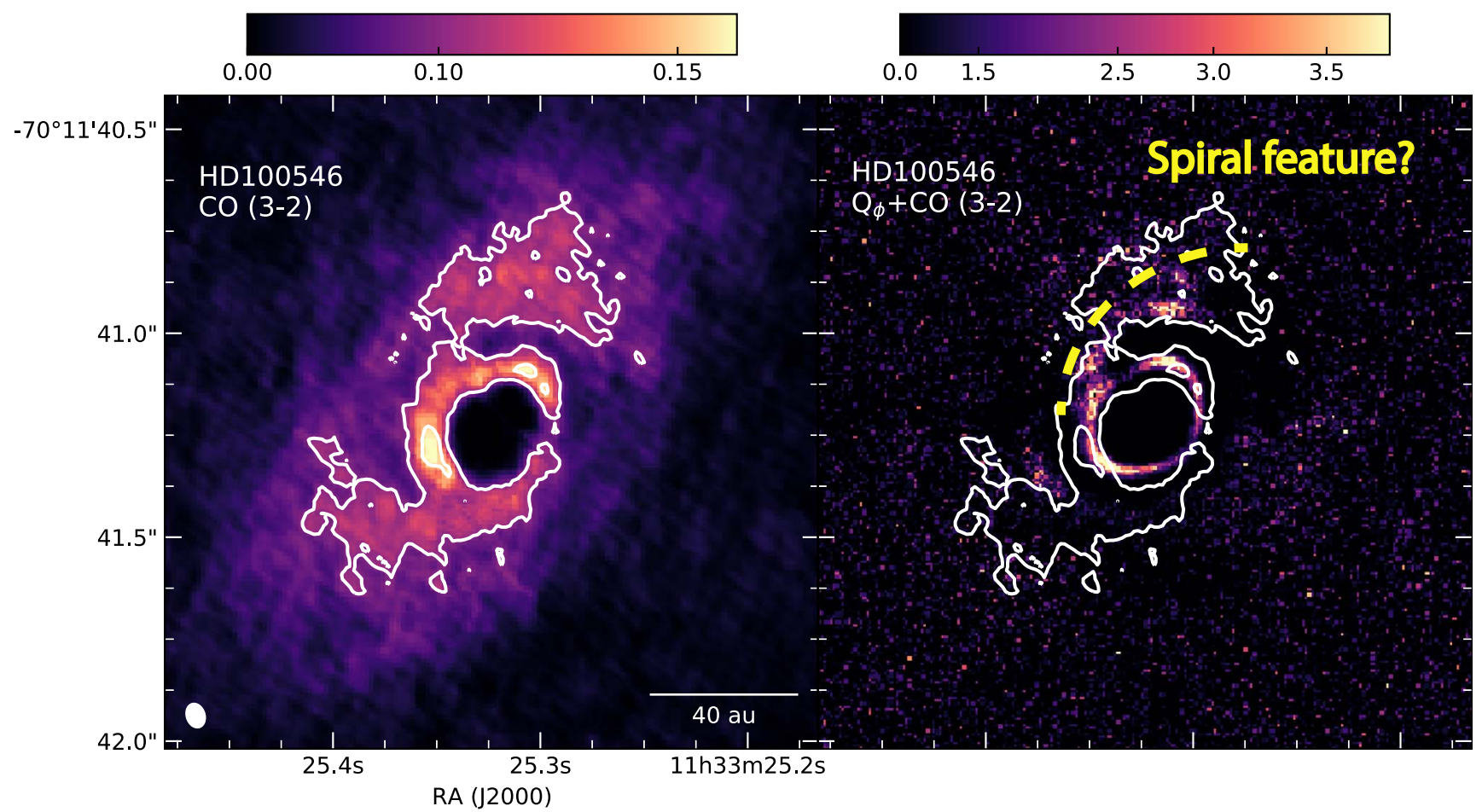

Figure 11. Comparison of CO-integrated intensity and unsharp masked version of a SPHERE/ZIMPOL $Q_{\phi}$ image. Left: background and contours show the integrated intensity map of $\mathrm{CO}$ (zoomed-in version of Figure 2) using a stretch to highlight the suggestive spiral-like emission highlighted by the contours. The bottom left and right corners show the beam and scale bar, respectively. Right: unsharp masked version of a SPHERE/ZIMPOL $Q_{\phi}$ image (as in Figure 10) overlaid with CO-integrated intensity contours shown in the left panel. The position and general orientation of the NE-arm feature seen with SPHERE is similar to the CO enhancement shown in the left panel. This would suggest the presence of a real spiral-like feature in the disk surface.

\section{Summary}

We presented new ALMA high angular resolution observations of the $870 \mu \mathrm{m}$ dust continuum and $\mathrm{CO}(3-2)$ of $\mathrm{HD}$ 100546. Our results can be summarized as follows:

1. The ALMA $870 \mu \mathrm{m}$ dust continuum and $\mathrm{CO}(3-2)$ observations achieve $\approx 50$ mas resolution, and they resolve the disk emission with unprecedented detail.

2. The continuum disk emission is resolved as ring-like (between 20 and $40 \mathrm{au}$ ) and shows a flux asymmetry of $\approx 15 \%-25 \%$.

3. The disk continuum emission is well fit by two concentric Gaussian rings plus a Gaussian vortex to reproduce the flux asymmetry; this morphology is similar to those of other disks.

4. Radial cuts show that the disk continuum profiles are well fit using a superposition of multiple-Gaussian profiles exemplifying the need for two Gaussian rings to match the two broader and narrower components of the main ring.

5. We searched for CPD emission at the location of the embedded planet candidate HD $100546 \mathrm{~b}$, but no pointlike continuum emission is detected. This places strong constraints on the CPD mass of $1.44 M_{\oplus}$ and radius of 0.44 au in the optically thin and thick cases, respectively.

6. The CPD mass upper limit is low enough to be incompatible with several planet accretion models, while synthetic observations of numerical simulations by Szulágyi et al. (2018) provide a CPD flux similar to the upper limit reported here. Gas-starved models are also still compatible.

7. We derive an upper limit on the planetary mass of $1.65 M_{J}$ based on a numerically calibrated relationship between CSD, $\mathrm{CPD}$, and planetary masses, assuming on-going accretion.
8. A central compact emission is also detected that arises from the inner central disk. We estimate an inner disk mass of $15 M_{\oplus}$, and using a previously estimated accretion rate onto the central star, we calculate an inner disk lifetime of $500 \mathrm{yr}$. Therefore, the inner disk must be replenished with material from the outer ring.

9. We compare high angular resolution SPHERE polarization data with ALMA continuum and CO emission. This suggest that the NE-arm feature seen in the polarized emission does not have a corresponding dust column density feature; however, it is well matched by a spirallike feature seen as enhanced CO-integrated emission. This is consistent with the expectation the both $\mathrm{CO}$ and small dust particles trace the disk surface.

J.E.P. acknowledges the financial support of the European Research Council (ERC; project PALs 320620). J.Sz. acknowledges the support from the Swiss National Science Foundation (SNSF) Ambizione grant PZ00P2_174115. S.P.Q. acknowledges the financial support of the SNSF. Parts of this work have been carried out within the framework of the National Center for Competence in Research PlanetS supported by the SNSF. F.M. acknowledges support from The Leverhulme Trust, the Isaac Newton Trust and the Royal Society Dorothy Hodgkin Fellowship. This paper makes use of the following ALMA data: ADS/JAO.ALMA\#2015.1.00806.S. ALMA is a partnership of ESO (representing its member states), NSF (USA) and NINS (Japan), together with NRC (Canada), NSC, and ASIAA (Taiwan), and KASI (Republic of Korea), in cooperation with the Republic of Chile. The Joint ALMA Observatory is operated by ESO, AUI/NRAO, and NAOJ. The National Radio Astronomy Observatory is a facility of the 
National Science Foundation operated under cooperative agreement by Associated Universities, Inc. This research made use of Astropy, a community-developed core Python package for Astronomy (Astropy Collaboration et al. 2013), matplotlib (Hunter 2007) and APLpy, an open-source plotting package for Python hosted at http://aplpy.github.com.

Facility: ALMA.

Software: Astropy (Astropy Collaboration et al. 2013), Matplotlib (Hunter 2007), CASA (McMullin et al. 2007), GALARIO (Tazzari et al. 2018).

\section{ORCID iDs}

Jaime E. Pineda (1) https://orcid.org/0000-0002-3972-1978 Judit Szulágyi (1) https://orcid.org/0000-0001-8442-4043 Sascha P. Quanz (i) https://orcid.org/0000-0003-3829-7412 Ewine F. van Dishoeck (1) https://orcid.org/0000-0001-7591-1907 Antonio Garufi (i) https://orcid.org/0000-0002-4266-0643 Farzana Meru (ib https://orcid.org/0000-0002-3984-9496 Gijs D. Mulders (10 https://orcid.org/0000-0002-1078-9493 Leonardo Testi (i) https://orcid.org/0000-0003-1859-3070 Michael R. Meyer (10 https://orcid.org/0000-0003-1227-3084 Maddalena Reggiani $\odot$ https://orcid.org/0000-0003-2911-0898

\section{References}

Acke, B., \& van den Ancker, M. E. 2006, A\&A, 449, 267

Andrews, S. M., Wilner, D. J., Espaillat, C., et al. 2011, ApJ, 732, 42

Astropy Collaboration, Robitaille, T. P., Tollerud, E. J., et al. 2013, A\&A, 558, A33

Ayliffe, B. A., \& Bate, M. R. 2012, MNRAS, 427, 2597

Beckwith, S. V. W., Sargent, A. I., Chini, R. S., \& Guesten, R. 1990, AJ, 99, 924

Benisty, M., Tatulli, E., Ménard, F., \& Swain, M. R. 2010, A\&A, 511, A75

Bergner, J. B., Guzmán, V. G., Öberg, K. I., Loomis, R. A., \& Pegues, J. 2018, ApJ, 857, 69

Biller, B. A., Liu, M. C., Wahhaj, Z., et al. 2013, ApJ, 777, 160

Biller, B. A., Males, J., Rodigas, T., et al. 2014, ApJL, 792, L22

Boss, A. P. 2001, ApJ, 563, 367

Bouwman, J., de Koter, A., Dominik, C., \& Waters, L. B. F. M. 2003, A\&A, 401, 577

Brittain, S. D., Najita, J. R., \& Carr, J. S. 2009, ApJ, 702, 85

Brittain, S. D., Najita, J. R., Carr, J. S., et al. 2013, ApJ, 767, 159

Bruderer, S., van Dishoeck, E. F., Doty, S. D., \& Herczeg, G. J. 2012, A\&A, 541, A91

Bryan, M. L., Knutson, H. A., Howard, A. W., et al. 2016, ApJ, 821, 89

Calcutt, H., Jørgensen, J. K., Müller, H. S. P., et al. 2018, A\&A, 616, A90

Canup, R. M., \& Ward, W. R. 2002, AJ, 124, 3404

Canup, R. M., \& Ward, W. R. 2006, Natur, 441, 834

Casassus, S., Wright, C. M., Marino, S., et al. 2015, ApJ, 812, 126

Chauvin, G., Desidera, S., Lagrange, A.-M., et al. 2017, A\&A, 605, L9

Chauvin, G., Lagrange, A.-M., Bonavita, M., et al. 2010, A\&A, 509, A52

Chauvin, G., Vigan, A., Bonnefoy, M., et al. 2015, A\&A, 573, A127

Cumming, A., Butler, R. P., Marcy, G. W., et al. 2008, PASP, 120, 531

Currie, T., Brittain, S., Grady, C. A., Kenyon, S. J., \& Muto, T. 2017, RNAAS, 1,40

Currie, T., Cloutier, R., Brittain, S., et al. 2015, ApJL, 814, L27

Currie, T., Muto, T., Kudo, T., et al. 2014, ApJL, 796, L30

de Val-Borro, M., Artymowicz, P., D’Angelo, G., \& Peplinski, A. 2007, A\&A, 471,1043

Fairlamb, J. R., Oudmaijer, R. D., Mendigutía, I., Ilee, J. D., \& van den Ancker, M. E. 2015, MNRAS, 453, 976

Fedele, D., Bruderer, S., van den Ancker, M. E., \& Pascucci, I. 2015, ApJ, 800,23

Follette, K. B., Rameau, J., Dong, R., et al. 2017, AJ, 153, 264

Folsom, C. P., Bagnulo, S., Wade, G. A., et al. 2012, MNRAS, 422, 2072

Friesen, R. K., Pineda, J. E., Rosolowsky, E., et al. 2017, ApJ, 843, 63

Gaia Collaboration, Brown, A. G. A., Vallenari, A., et al. 2018, A\&A, 616, A1

Garufi, A., Quanz, S. P., Schmid, H. M., et al. 2016, A\&A, 588, A8
Grady, C. A., Woodgate, B., Heap, S. R., et al. 2005, ApJ, 620, 470

Gressel, O., Nelson, R. P., Turner, N. J., \& Ziegler, U. 2013, ApJ, 779, 59

Hauschildt, P. H., Allard, F., \& Baron, E. 1999, ApJ, 512, 377

Heinze, A. N., Hinz, P. M., Sivanandam, S., et al. 2010, ApJ, 714, 1551

Helled, R., Bodenheimer, P., Podolak, M., et al. 2014, Protostars and Planets

VI (Tucson, AZ: Univ. Arizona Press), 643

Huang, P., Isella, A., Li, H., Li, S., \& Ji, J. 2018, ApJ, 867, 3

Hunter, J. D. 2007, CSE, 9, 90

Isella, A., Chandler, C. J., Carpenter, J. M., Pérez, L. M., \& Ricci, L. 2014, ApJ, 788, 129

Isella, A., Pérez, L. M., \& Carpenter, J. M. 2012, ApJ, 747, 136

Johnson, J. A., Aller, K. M., Howard, A. W., \& Crepp, J. R. 2010, PASP, 122,905

Keppler, M., Benisty, M., Müller, A., et al. 2018, A\&A, 617, A44

Kraus, A. L., \& Ireland, M. J. 2012, ApJ, 745, 5

Kraus, S., Kreplin, A., Fukugawa, M., et al. 2017, ApJL, 848, L11

Lafrenière, D., Doyon, R., Marois, C., et al. 2007, ApJ, 670, 1367

Lagrange, A.-M., Bonnefoy, M., Chauvin, G., et al. 2010, Sci, 329, 57

Lambrechts, M., \& Johansen, A. 2012, A\&A, 544, A32

Liskowsky, J. P., Brittain, S. D., Najita, J. R., et al. 2012, ApJ, 760, 153

Liu, W. M., Hinz, P. M., Meyer, M. R., et al. 2003, ApJL, 598, L111

Loomis, R. A., Öberg, K. I., Andrews, S. M., \& MacGregor, M. A. 2017, ApJ, 840,23

Lyra, W., \& Lin, M.-K. 2013, ApJ, 775, 17

Macintosh, B., Graham, J. R., Barman, T., et al. 2015, Sci, 350, 64

Marois, C., Macintosh, B., Barman, T., et al. 2008, Sci, 322, 1348

McMullin, J. P., Waters, B., Schiebel, D., Young, W., \& Golap, K. 2007, in ASP Conf. Ser. 376, Astronomical Data Analysis Software and Systems XVI, ed. R. A. Shaw, F. Hill, \& D. J. Bell (San Francisco, CA: ASP), 127 Meshkat, T., Kenworthy, M. A., Reggiani, M., et al. 2015, MNRAS, 453, 2533 Mittal, T., \& Chiang, E. 2015, ApJL, 798, L25

Mulders, G. D., Paardekooper, S.-J., Panić, O., et al. 2013, A\&A, 557, A68 Nielsen, E. L., Liu, M. C., Wahhaj, Z., et al. 2013, ApJ, 776, 4 Panić, O., \& Hogerheijde, M. R. 2009, A\&A, 508, 707

Panić, O., Ratzka, T., Mulders, G. D., et al. 2014, A\&A, 562, A101 Panić, O., van Dishoeck, E. F., Hogerheijde, M. R., et al. 2010, A\&A, 519, A110 Pérez, L. M., Isella, A., Carpenter, J. M., \& Chandler, C. J. 2014, ApJL, 783, L13 Perez, S., Dunhill, A., Casassus, S., et al. 2015, ApJL, 811, L5

Pineda, J. E., Quanz, S. P., Meru, F., et al. 2014, ApJL, 788, L34 Pinilla, P., Klarmann, L., Birnstiel, T., et al. 2016, A\&A, 585, A35 Pinilla, P., Pérez, L. M., Andrews, S., et al. 2017, ApJ, 839, 99 Pollack, J. B., Hubickyj, O., Bodenheimer, P., et al. 1996, Icar, 124, 62 Quanz, S. P., Amara, A., Meyer, M. R., et al. 2013, ApJL, 766, L1 Quanz, S. P., Amara, A., Meyer, M. R., et al. 2015, ApJ, 807, 64 Quanz, S. P., Schmid, H. M., Geissler, K., et al. 2011, ApJ, 738, 23 Quillen, A. C. 2006, ApJ, 640, 1078

Quillen, A. C., \& Trilling, D. E. 1998, ApJ, 508, 707

Rameau, J., Chauvin, G., Lagrange, A.-M., et al. 2013a, A\&A, 553, A60

Rameau, J., Chauvin, G., Lagrange, A.-M., et al. 2013b, ApJL, 772, L15

Rameau, J., Follette, K. B., Pueyo, L., et al. 2017, AJ, 153, 244

Reggiani, M., Christiaens, V., Absil, O., et al. 2018, A\&A, 611, A74 Reggiani, M., Meyer, M. R., Chauvin, G., et al. 2016, A\&A, 586, A147 Reggiani, M., Quanz, S. P., Meyer, M. R., et al. 2014, ApJL, 792, L23 Ricci, L., Cazzoletti, P., Czekala, I., et al. 2017, AJ, 154, 24

Sallum, S., Follette, K. B., Eisner, J. A., et al. 2015, Natur, 527, 342 Shabram, M., \& Boley, A. C. 2013, ApJ, 767, 63

Siess, L., Dufour, E., \& Forestini, M. 2000, A\&A, 358, 593

Sissa, E., Gratton, R., Garufi, A., et al. 2018, A\&A, 619, A160

Stamatellos, D., \& Herczeg, G. J. 2015, MNRAS, 449, 3432

Szulágyi, J. 2017, ApJ, 842, 103

Szulágyi, J., Mayer, L., \& Quinn, T. 2017, MNRAS, 464, 3158

Szulágyi, J., Morbidelli, A., Crida, A., \& Masset, F. 2014, ApJ, 782, 65 Szulágyi, J., Plas, G. v. d., Meyer, M. R., et al. 2018, MNRAS, 473, 3573

Tatulli, E., Benisty, M., Ménard, F., et al. 2011, A\&A, 531, A1

Tazzari, M., Beaujean, F., \& Testi, L. 2018, MNRAS, 476, 4527

van der Marel, N., van Dishoeck, E. F., Bruderer, S., et al. 2013, Sci, 340, 1199

van der Plas, G., van den Ancker, M. E., Acke, B., et al. 2009, A\&A, 500,1137

Wahhaj, Z., Liu, M. C., Nielsen, E. L., et al. 2013, ApJ, 773, 179

Walsh, C., Daley, C., Facchini, S., \& Juhász, A. 2017, A\&A, 607, A114

Walsh, C., Juhász, A., Pinilla, P., et al. 2014, ApJL, 791, L6

Wittenmyer, R. A., Butler, R. P., Tinney, C. G., et al. 2016, ApJ, 819, 28

Wright, C. M., Maddison, S. T., Wilner, D. J., et al. 2015, MNRAS, 453, 414

Zhu, Z., Ju, W., \& Stone, J. M. 2016, ApJ, 832, 193 